\title{
Comparing the effectiveness of ultrasound guided versus blind genicular nerve block on pain, muscle strength with isokinetic device, physical function and quality of life in chronic knee osteoarthritis: a prospective randomized controlled study
}

\author{
Damla Cankurtaran, Ozgur Zeliha Karaahmet, Sadik Yigit Yildiz, Emel Eksioglu, Deniz Dulgeroglu, and \\ Ece Unlu
}

Department of Physical Medicine and Rehabilitation, Diskapi Yildirim Beyazit Education and Research Hospital, Ankara, Turkey

Received February 3, 2020

Revised May 9, 2020

Accepted May 13, 2020

Handling Editor: Jin Woo Shin

\section{Correspondence}

Damla Cankurtaran

Department of Physical Medicine and

Rehabilitation, Diskapi Yildirim Beyazit

Education and Research Hospital, Ziraat

Distict Şehit Ömer Halisdemir Street,

Gate number: 20, Dışkapı, Ankara

06110, Turkey

Tel: +90-5054558101

Fax: +90-312-318-6690

E-mail: damlacengizftr@gmail.com
Background: The genicular nerve block (GNB) is demonstrated from several reports to alleviate pain and improve knee functionality in patients with chronic knee osteoarthritis (OA). Ultrasound (US)-guided GNB has been the most used imaging method. This study aimed to compare the effectiveness of US-guided versus blind GNB in the treatment of knee OA.

Methods: This prospective, randomized clinical trial included patients with knee OA based on American College of Rheumatology diagnostic criteria. The patients were evaluated for clinical and dynamometer parameters at the baseline, 4 weeks after treatment, and 12 weeks after treatment. The patients underwent blind injection or US-guided injection.

Results: When compared with the baseline, both groups showed significant improvement in pain, physical function, and quality of life parameters. Significant differences were observed between the groups for clinical parameters (30-second chair stand test, 6-minute walk test) in favor of the US-guided group. On the other hand, blind injection was more significantly effective on some parameters of the Nottingham Health Profile. There wasn't any significant improvement in isokinetic muscle strength for either group.

Conclusions: This study demonstrated that both US-guided and blind GNB, in the treatment of knee $\mathrm{OA}$, were effective in reducing symptoms and improving physical function. GNB wasn't an effective treatment for isokinetic muscle function. USguided injections may yield more effective clinical results than blind injections.

Key Words: Knee Joint; Muscle Strength; Nerve Block; Osteoarthritis, Knee; Pain Management; Quality of Life; Steroids; Treatment Outcome; Ultrasonography, Interventional.

\section{INTRODUCTION}

Osteoarthritis (OA) is one of the most common degenerative joint diseases among elderly individuals, and knee joints are the most affected joints [1,2]. Pain, joint stiffness, and decreased muscle strength can be seen, and cause poor quality of life and poor functional capacity [3]. Conservative pharmacological treatments such as oral analge- (a) This is an open-access article distributed under the terms of the Creative Commons Attribution Non-Commercial License (http://creativecommons.org/licenses/by-nc/4.0/), which permits unrestricted non-commercial use, distribution, and reproduction in any medium, provided the original work is properly cited.

(C) The Korean Pain Society, 2020
Author contributions: Damla Cankurtaran: Writing/manuscript preparation; Ozgur Zeliha Karaahmet: Writing/manuscript preparation; Sadik Yigit Yildiz: Data curation; Emel Eksioglu: Study conception; Deniz Dulgeroglu: Study conception; Ece Unlu: Supervision. 
sics, viscosupplementation, intraarticular corticosteroid injections, acupuncture, and prolotherapy, as well as nonpharmacological treatments, may be inadequate [4]. When conservative treatment fails, total knee joint arthroplasty may be an option; however, total knee arthroplasty can't be performed on all patients because some patients have comorbidities and the risk of surgery complications [5].

Genicular nerve block (GNB) is demonstrated from several reports to alleviate pain and improve knee functionality in patients with chronic knee OA [6-8]. Ultrasound (US) has been the most used imaging to aid in landmark targeting and procedural accuracy in recent studies. The genicular nerves are the main innervating articular branches for the knee joint, and as they are adjacent to the periosteum, connecting the bone, they can be located using bony landmarks. Superomedial, inferomedial, and superolateral genicular nerve (SMGN, IMGN, and SLGN) branches have been targeted for these treatment options in previous studies [7,9]. The location of these nerves, their anatomical relationship with surrounding tissues, and their origin and termination become better understood through cadaveric studies; therefore, it is possible to perform GNB without imaging [10,11].

Studies on the efficacy of the GNB on knee OA have generally focused on the effect of pain, because pain is the key symptom of knee OA [12]. The efficacy of GNB on thigh muscle strength, functional level, and quality of life hasn't been examined, and blind GNB hasn't been compared to US-guided GNB in previous studies. Therefore, the aim of this study is to compare the efficiency and safety of USguided versus blind GNB on pain, physical function, quality of life, and isokinetic muscle performance in patients with chronic knee OA.

\section{MATERIALS AND METHODS}

\section{Study design}

This prospective, randomized clinical trial evaluated patients at the Department of Physical Medicine and Rehabilitation, Diskapi Yildirim Beyazit Education and Research Hospital that were treated with a GNB using either US-guided or blind GNB techniques for knee OA, according to the American College of Rheumatology diagnostic criteria [13], between January 2019 and April 2019. The study protocol was approved by the hospital's local ethics committee (approval number 72/05). The protocol was explained to all patients, and informed consent was obtained at the beginning of the study.

\section{Patients}

The patients included in this study had chronic knee OA with more than 3 months of pain, a radiological OA grade greater than Kellgren-Lawrence (KL) grade 2 [14], 50-80 years of age, and refractory knee OA pain not alleviated with analgesics.

The patients with acute knee pain (less than 3 mo); connective tissue diseases affecting the knee; serious neurological or psychiatric disorders; history of trauma; surgery to the knee joint; steroid/hyaluronic acid injection; physical or exercise therapy over the past 3 months; cardiovascular, respiratory, metabolic, blood, thyroid, rheumatic disease, or malignancy; genetic bone/joint disease; morbid obesity ( $>35 \mathrm{~kg} / \mathrm{mm}^{2}$ ); or prior use of an anticoagulant were excluded. The patients were randomly assigned by a computer-generated randomization schedule into two groups: a blind GNB group and a US-guided GNB group. The injections were performed by two physicians; all injections were performed by the same physicians, who were blind to the patients' evaluations within the groups.

The sample size was made using the $\mathrm{G}^{*}$ power (V3.1.7) program (available freely at https://www.psychologie. hhu.de/arbeitsgruppen/allgemeine-psychologie-und-arbeitspsychologie/gpower.html). In order to create a minimum change of 10 units in the visual analogue scale (VAS), at least 20 patients were found for each group with $\alpha=0.05$, $80 \%$ power, and $d=0.631$ for the effect size. The sample size is also compatible with similar previous studies [15].

\section{GNB techniques}

After the patients in group 1 were placed in a supine position with a pillow under the popliteal fossa to provide comfort, the US-guided injection technique was similar to techniques in previous studies $[3,15,16]$. The volume totaled $6 \mathrm{~mL}$ which was comprised of $2 \%$ lidocaine and 20 $\mathrm{mg}$ of triamcinolone, which was divided between the three injection sites.

A cadaveric study showed that the SMGN curves around the femur shaft and goes to the area between the adductor magnus tendon and the femoral medial epicondyle; then it travels about one $\mathrm{cm}$ anterior to the adductor tubercle. The IMGN is another branch of the tibial nerve that separates in the inferior popliteal region and is settled horizontally around the lower parts of the tibial medial epicondyle [10]. Another cadaveric study indicated that the SLGN travels directly to the synovial surface of the lateral femoral epicondyle [11]. Based on these studies, we decided to describe three lines to determine the injection points for the procedure without imaging.

After the patients in group 2 were placed in supine posi- 


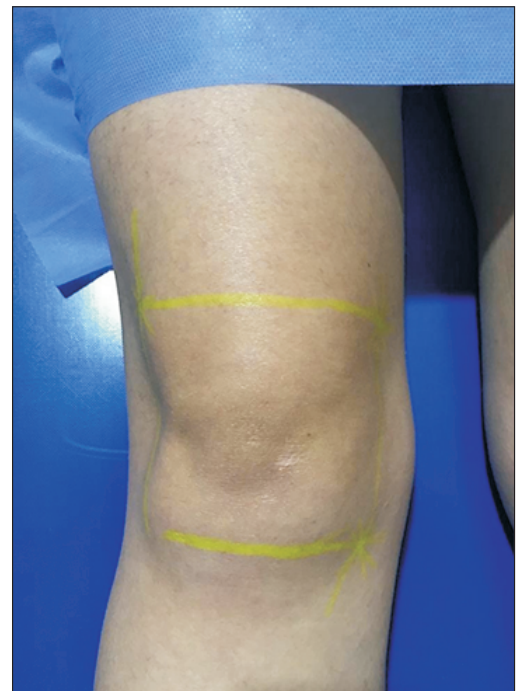

Fig. 1. The lines were used for blind injection. Blind injection was applied at superolateral, superomedial and inferomedial corners of the lines.

tion with full knee extension, a line was drawn longitudinally through the fibular head extending superiorly along the femur to a level $4 \mathrm{~cm}$ superior to the tip of the lateral femoral epicondyle; another line was ruled horizontally between the femur epicondyles, and the third line was crossed from the femur medial epicondyle to the tibial medial epicondyle (Fig. 1). After needle was inserted in the first, second, and third points to the deep of the periosteum, the needle was retracted and the injection was applied. Before the injection, sonographic evaluation was made of the US-guided injection group, while the blind group was not evaluated.

\section{Outcome parameters}

Another physician, who was blinded to the treatment allocations, evaluated all measurements (baseline, after $4 \mathrm{wk}$, and after $12 \mathrm{wk}$ ). Demographic data such as age, gender, and body mass index (BMI) were recorded. Weight-bearing radiographs were reviewed at baseline, and the KL system was used to grade the degree of OA [14].

Pain intensity was assessed using the $100 \mathrm{~mm}$ VAS. The Western Ontario and McMaster Universities Osteoarthritis Index (WOMAC) was used to measure subjective knee functionality. The WOMAC consists of 24 questions (5 pain, 2 joint stiffness, and 17 functional limitation questions). All questions are scored on a scale of 0 to 4 . The total scores for pain, stiffness, physical function, and total, which ranged from 0 to 20,0 to 8,0 to 68 , and 0 to 96 respectively, were then determined [17]. The Nottingham Health Profile (NHP) was applied to each participant to determine the level of quality of life. The NHP is a selfadministered questionnaire that is used to determine and quantify perceived health problems. It is divided into six subscales (sleep, mobility, energy, pain, emotional reactions, and social isolation) and consists of 38 items [18]. The Timed Up and Go Test (TUGT) [19], 6-minute walk test (6MWT) [20], 30-second chair stand test (30-s CST) [21], and stair climb test (SCT) [22] were done to evaluate functional mobility. The 6MWT was performed in a 30-meterlong corridor, and measured the total distance walked in meters over 6 minutes [23]. The TUGT involves the patient getting up from a chair, walking 3 meters, and returning to sit in the same chair. After the patient completed the test we noted the completion time [23]. The 30-s CST is a test that counts the total number of complete chair stands within 30 seconds [23]. The SCT measures the time to ascend and descend 12 stairs with an 18-cm step height. Complications and pain during injection (VAS 0-100 mm) were inquired about at the second visit.

Biodex System 3 Dynamometer (Biodex ${ }^{\circledR}$ Corp., Shirley, $\mathrm{NY}$ ) was used to measure the isokinetic muscle strength of the quadriceps and hamstrings. The performance of the muscles of the affected knee was evaluated with isokinetic tests in all patients in both groups before treatment, 4 weeks after treatment, and 12 weeks after treatment. During the test, angular velocities of $60 \% \mathrm{sec}$ and $180 \% \mathrm{sec}$ were preferred as being commonly used angular velocities. The isokinetic test protocol was applied for the first 3 submaximal warm-up repetitions, and after 5 and 10 maximal effort repetitions with a 10 -second rest period between each repetition at $60 \% \mathrm{sec}$ and $180 \% \mathrm{sec}$ angular velocity. Isokinetic knee extensor and flexor mean peak torque (PT) was determined, and was standardized to body weight. All tests were performed by one observer.

\section{Statistical analysis}

Statistical analysis was performed using IBM SPSS ver. 20.0 (IBM Corp., Armonk, NY). The Shapiro-Wilk test was used to analyze whether the continuous variables were normally distributed. Normally distributed demographic data were compared using an independent sample $t$-test and presented as mean \pm standard deviation. Nonparametric data were evaluated using Mann-Whitney $U$-tests, and presented as median and interquartile range. Categorical data were presented as numbers and percentages and compared using the chi-square test or Fisher's exact test. For $P<0.05$, the results were considered significant. Freidman and Wilcoxon tests were used to evaluate whether the variations between the repetitive measurements were significant. Bonferroni correction was used to avoid possible type 1 errors, and $P<0.017$ values were accepted as statistically significant. 
Table 1. Comparison Changes of VAS and WOMAC Scores

\begin{tabular}{|c|c|c|c|c|}
\hline Variable & & US-guided GNB & Blind GNB & $P$ value \\
\hline \multirow[t]{3}{*}{ VAS } & 1 mo-baseline & $-32.0 \pm 6.1$ & $-26.0 \pm 5.5$ & 0.58 \\
\hline & 3 mo-1 mo & $12.0 \pm 7.4$ & $8.8 \pm 5.1$ & 0.78 \\
\hline & 3 mo-baseline & $-20.0 \pm 4.5$ & $-17.3 \pm 5.8$ & 0.43 \\
\hline \multirow[t]{3}{*}{ WOMAC-pain } & 1 mo-baseline & $-1.25 \pm 0.72$ & $-2.11 \pm 0.96$ & 0.39 \\
\hline & 3 mo-1 mo & $0.35 \pm 0.57$ & $-0.61 \pm 1.24$ & 0.82 \\
\hline & 3 mo-baseline & $-0.91 \pm 0.49$ & $-2.71 \pm 1.28$ & 0.36 \\
\hline \multirow[t]{3}{*}{ WOMAC-stiffness } & 1 mo-baseline & $-1.26 \pm 0.39$ & $-0.56 \pm 0.33$ & 0.11 \\
\hline & 3 mo-1 mo & $-0.15 \pm 0.48$ & $0.11 \pm 0.35$ & 0.71 \\
\hline & 3 mo-baseline & $-1.41 \pm 0.41$ & $-0.46 \pm 0.45$ & 0.14 \\
\hline \multirow[t]{3}{*}{ WOMAC-physical function } & 1 mo-baseline & $-6.51 \pm 3.18$ & $-7.31 \pm 2.29$ & 0.59 \\
\hline & $3 \mathrm{mo-1}$ mo & $2.01 \pm 3.37$ & $-0.61 \pm 4.19$ & 0.32 \\
\hline & 3 mo-baseline & $-4.51 \pm 3.09$ & $-7.9 \pm 2.86$ & 0.18 \\
\hline \multirow[t]{3}{*}{ WOMAC-total } & 1 mo-baseline & $-9.11 \pm 3.95$ & $-9.96 \pm 3.21$ & 0.81 \\
\hline & $3 \mathrm{mo-1}$ mo & $0.11 \pm 4.62$ & $-1.26 \pm 5.42$ & 0.83 \\
\hline & 3 mo-baseline & $-9.01 \pm 3.01$ & $-14.21 \pm 3.87$ & 0.81 \\
\hline
\end{tabular}

Values are presented as mean \pm standard deviation.

VAS: visual analogue scale, WOMAC: Western Ontario and McMaster's Universities Osteoarthritis Index, US: ultrasound, GNB: genicular nerve block.

Table 2. Comparison Changes of NHP

\begin{tabular}{llccc}
\hline Variable & & US-guided GNB & Blind GNB & $P$ value \\
\hline NHP-pain & 1 mo-baseline & $-19.75 \pm 2.74$ & $-12.84 \pm 4.03$ & 0.13 \\
& 3 mo-1 mo & $9.94 \pm 4.16$ & $-5.29 \pm 4.49$ & 0.19 \\
NHP-emotional reaction & 3 mo-baseline & $-9.82 \pm 3.85$ & $-18.12 \pm 4.28$ & $0.03^{*}$ \\
& 1 mo-baseline & $9.28 \pm 5.07$ & $-5.36 \pm 4.89$ & 0.09 \\
NHP-sleep & 3 mo-1 mo & $2.26 \pm 3.23$ & $-4.16 \pm 4.72$ & 0.35 \\
& 3 mo-baseline & $11.55 \pm 6.49$ & $-9.51 \pm 3.50$ & $0.03^{*}$ \\
NHP-social isolation & 1 mo-baseline & $-5.61 \pm 6.12$ & $-0.64 \pm 2.14$ & 0.49 \\
& 3 mo-1 mo & $0.91 \pm 5.76$ & $11.09 \pm 7.53$ & 0.35 \\
NHP-physical mobility & 3 mo-baseline & $-4.73 \pm 5.82$ & $10.46 \pm 7.27$ & $0.01 *$ \\
& 1 mo-baseline & $10.83 \pm 3.23$ & $-9.91 \pm 6.37$ & 0.24 \\
NHP-energy & 3 mo-1 mo & $-1.46 \pm 1.72$ & $-5.13 \pm 6.69$ & 0.09 \\
& 3 mo-baseline & $9.38 \pm 3.11$ & $-15.04 \pm 5.72$ & 0.09 \\
& 1 mo-baseline & $-12.01 \pm 5.26$ & $-6.14 \pm 3.20$ & 0.44 \\
& 3 mo-1 mo & $-2.03 \pm 3.13$ & $5.74 \pm 4.10$ & 0.08 \\
& 3 mo-baseline & $-9.98 \pm 4.34$ & $-0.41 \pm 3.54$ & 0.45 \\
\hline
\end{tabular}

Values are presented as mean \pm standard deviation.

NHP: Nottingham Health Profile, US: ultrasound, GNB: genicular nerve block.

*Asterisk indicates a statistically significant $(P<0.05)$.

\section{RESULTS}

Twenty-three patients (11 patients and 20 knees in the USguided injection group; 12 patients and 20 knees in the blind injection group) were enrolled in the present study. Nine patients in the US-guided injection group, and 8 patients in the blind injection group, underwent a bilateral GNB. The other 7 patients (3 patients in the US-guided injection group and 4 patients in the blind injection group) underwent GNB to only one side. The groups were similar in terms of findings at the baseline. No significant differences were determined between the groups in terms of age, BMI, KL grade, VAS pain, WOMAC, SCT, 30-s CST,
TUGT, 6MWT, and isokinetic test values $(P>0.05)$. Only the NHP emotional reactions and social isolation subscales had a significant difference between groups $(P=$ $0.03, P=0.02$ ).

A comparison of changes in the VAS, WOMAC, and NHP from baseline to first month, third month to first month, and third month to baseline, between groups are shown in Table 1 and Table 2. According to a comparison of the first month to baseline, the significant difference was determined to be the social isolation subscale of the NHP between the groups $(P=0.01)$. The subscales pain and emotional reaction of the NHP were significantly changed at the third month compared to the baseline between the 
Table 3. Comparison Changes of Clinical Functional Test and Isokinetic Test

\begin{tabular}{|c|c|c|c|c|}
\hline Variable & & US-guided GNB & Blind GNB & $P$ value \\
\hline \multirow[t]{3}{*}{ SCT } & 1 mo-baseline & $-2.65 \pm 0.48$ & $-1.57 \pm 0.45$ & 0.11 \\
\hline & 3 mo-1 mo & $1.06 \pm 0.35$ & $0.75 \pm 0.26$ & 0.01 \\
\hline & 3 mo-baseline & $-1.60 \pm 0.41$ & $-0.82 \pm 0.42$ & 0.31 \\
\hline \multirow[t]{3}{*}{ TUGT } & 1 mo-baseline & $-2.16 \pm 0.29$ & $-1.16 \pm 0.34$ & 0.06 \\
\hline & 3 mo-1 mo & $0.13 \pm 0.26$ & $0.36 \pm 0.18$ & 0.62 \\
\hline & 3 mo-baseline & $-2.04 \pm 0.39$ & $-0.79 \pm 0.34$ & 0.07 \\
\hline \multirow[t]{3}{*}{ 30-s CST } & 1 mo-baseline & $1.91 \pm 0.27$ & $0.16 \pm 0.39$ & $0.01 *$ \\
\hline & 3 mo-1 mo & $-1.15 \pm 0.13$ & $0.05 \pm 2.66$ & $0.01 *$ \\
\hline & 3 mo-baseline & $0.75 \pm 0.23$ & $0.21 \pm 0.31$ & 0.31 \\
\hline \multirow[t]{3}{*}{ 6MWT } & 1 mo-baseline & $41.31 \pm 4.99$ & $0.46 \pm 23.49$ & $0.01 *$ \\
\hline & 3 mo-1 mo & $-29.31 \pm 3.89$ & $5.01 \pm 20.47$ & 0.53 \\
\hline & 3 mo-baseline & $12.01 \pm 6.69$ & $5.46 \pm 9.65$ & 0.08 \\
\hline \multirow[t]{3}{*}{ PT-Quadriceps $60^{\circ} / \mathrm{sec}$} & 1 mo-baseline & $0.28 \pm 2.91$ & $-3.47 \pm 3.65$ & 0.45 \\
\hline & $3 \mathrm{mo}-1 \mathrm{mo}$ & $28.46 \pm 28.58$ & $5.38 \pm 2.32$ & 0.19 \\
\hline & 3 mo-baseline & $28.73 \pm 28.89$ & $1.91 \pm 3.22$ & 0.91 \\
\hline \multirow[t]{3}{*}{ PT-Quadriceps $180^{\circ} / \mathrm{sec}$} & 1 mo-baseline & $0.24 \pm 1.45$ & $-6.26 \pm 3.23$ & 0.21 \\
\hline & 3 mo-1 mo & $1.59 \pm 1.65$ & $3.21 \pm 1.32$ & 0.07 \\
\hline & 3 mo-baseline & $1.83 \pm 1.56$ & $-3.05 \pm 3.59$ & 0.59 \\
\hline \multirow[t]{3}{*}{ PT-Hamstring $-60^{\circ} / \mathrm{sec}$} & 1 mo-baseline & $-1.79 \pm 2.04$ & $1.67 \pm 1.47$ & 0.51 \\
\hline & $3 \mathrm{mo}-1 \mathrm{mo}$ & $-0.97 \pm 1.03$ & $2.64 \pm 1.48$ & $0.03 *$ \\
\hline & 3 mo-baseline & $-2.76 \pm 2.01$ & $4.29 \pm 2.27$ & $0.01 *$ \\
\hline \multirow[t]{3}{*}{ PT-Hamstring $-180^{\circ} / \mathrm{sec}$} & 1 mo-baseline & $-0.21 \pm 1.21$ & $-0.88 \pm 2.32$ & 0.26 \\
\hline & $3 \mathrm{mo}-1 \mathrm{mo}$ & $0.94 \pm 1.39$ & $0.48 \pm 2.22$ & 0.51 \\
\hline & 3 mo-baseline & $0.73 \pm 0.77$ & $0.41 \pm 1.27$ & 0.74 \\
\hline
\end{tabular}

Values are presented as mean \pm standard deviation.

US: ultrasound, GNB: genicular nerve block, SCT: stair climb test, TUGT: timed up and go test, 30-s CST: 30-second chair stand test, 6MWT: 6-minute walk test, PT: peak torque.

*Asterisk indicates a statistically significant $(P<0.05)$.

groups ( $P=0.03$ and $P=0.03$, respectively).

Table 3 shows the comparison of the clinical functional test and isokinetic test from baseline to first month, third month to first month, and third month to baseline between the groups. There was a significant improvement in the 30-s CST from the first month to baseline $(P=0.01)$; but the SCT and the 30-s CST were significantly regressed after the first month $(P=0.01$ and $P=0.01$, respectively). The patients in the US-guided injection group exhibited significantly better performance in the 6MWT at the first month. The PT of the hamstring at $60 \% \mathrm{sec}$ was significantly different from the third month to first month and baseline in the blind injection group compared to the USguided injection group ( $P=0.03$ and $P=0.01$, respectively).

Fig. 2 showed the change in three months regarding the VAS, WOMAC, and NHP in the US-guided injection group and blind injection group. Changes in isokinetic test values at three months were presented in Fig. 3. In the US-guided injection group VAS, WOMAC-stiffness, NHPpain, SCT, TUGT, 30-s CST, and 6MWT were significant development after one month to injection $(P<0.017)$ (Table 4, Fig. 2A). Similarly, patients in the blind injection group had significantly better results for VAS, WOMAC-physical function, WOMAC-total, NHP-pain, SCT, and TUGT $(P<$ 0.017) (Table 4, Fig. 2B). Regarding the 3-month follow- up results compared with the baseline, VAS, WOMACstiffness, WOMAC-total, SCT, TUGT, and 30-s CST results significantly progressed in the US-guided injection group $(P<0.017)$ (Table 4, Fig. 2A). In the blind injection group, VAS, WOMAC-physical function, WOMAC-total and NHPpain, NHP-emotion reaction, and NHP-social isolation scores had significantly advancement three months after injection compared to the baseline $(P<0.017)$ (Table 4, Fig. 2B).

Comparing the 3-month results with the 1-month results, the patients in group 1 showed significantly worse performance with the SCT, 30-s CST, and 6MWT $(P<0.017)$ (Table 4, Fig. 2A). Similar to patients in the US-guided injection group, at three months the patients in the blind injection group were significantly slower compared to at one month regarding the SCT $(P=0.002)$ (Table 4 , Fig. 2B). Accordingly, within-group analyses of isokinetic test values showed there was no significant difference between the 1-month and 3-month results comparing the baseline, or the 3 -month results comparing the 1-month results $(P$ $>0.017$ ) (Fig. 3). There weren't any injection-related side effects in either group. Suprapatellar effusion was examined in five patients in the blind injection group. In the US-guided injection group the patients felt significant less pain compared to group 2 during the injection $(P=0.01)$. 

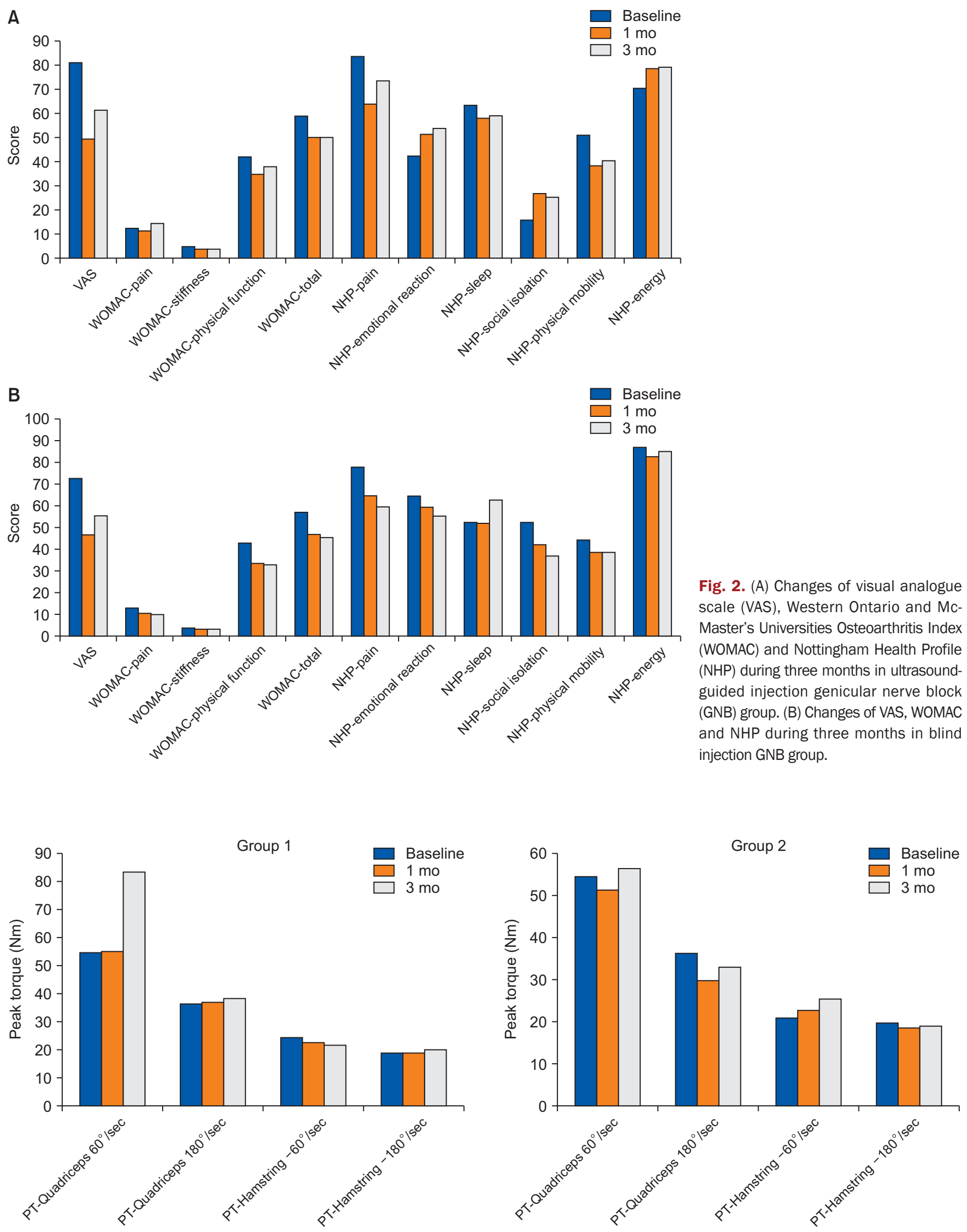

Fig. 3. Changes of isokinetic test results during three months within groups. PT: peak torque. 
Table 4. Change in Three Months the Results of the Clinical Functional Test

\begin{tabular}{|c|c|c|c|c|c|c|c|}
\hline Group & & Baseline & $1 \mathrm{mo}$ & $3 \mathrm{mo}$ & $\begin{array}{c}1 \text { mo-baseline } \\
P \text { value }\end{array}$ & $\begin{array}{c}3 \text { mo-1 mo } \\
P \text { value }\end{array}$ & $\begin{array}{c}3 \text { mo-baseline } \\
P \text { value }\end{array}$ \\
\hline \multirow[t]{4}{*}{ US-guided GNB } & SCT & $16.16 \pm 4.77$ & $13.51 \pm 3.58$ & $14.57 \pm 4.56$ & $0.001 *$ & $0.011 *$ & $0.002 *$ \\
\hline & TUGT & $11.58 \pm 2.52$ & $9.41 \pm 1.76$ & $9.54 \pm 2.15$ & $0.001 *$ & 0.82 & $0.001 *$ \\
\hline & 30-s CST & $8.71 \pm 2.25$ & $10.61 \pm 2.52$ & $9.45 \pm 2.64$ & $0.001 *$ & $0.001 *$ & $0.007 *$ \\
\hline & 6MWT & $364.71 \pm 71.74$ & $406 \pm 65.71$ & $376 \pm 60.58$ & $0.001 *$ & 0.58 & $0.001 *$ \\
\hline \multirow[t]{4}{*}{ Blind GNB } & SCT & $13.72 \pm 2.72$ & $12.16 \pm 3.06$ & $12.90 \pm 2.75$ & $0.002 *$ & 0.02 & 0.03 \\
\hline & TUGT & $10.09 \pm 1.89$ & $8.94 \pm 1.21$ & $9.25 \pm 1.04$ & $0.004 *$ & 0.03 & 0.04 \\
\hline & 30-s CST & $9.95 \pm 2.43$ & $10.11 \pm 2.95$ & $10.15 \pm 2.03$ & 0.71 & 0.71 & 0.45 \\
\hline & 6MWT & $400.75 \pm 62.53$ & $401 \pm 89.44$ & $406.21 \pm 27.41$ & 0.07 & $0.011 *$ & 0.31 \\
\hline
\end{tabular}

Values are presented as mean \pm standard deviation.

US: ultrasound, GNB: genicular nerve block, SCT: stair climb test, TUGT: timed up and go test, 30-s CST: 30-second chair stand test, 6MWT: 6-minute walk test.

*Asterisk indicates a statistically significant $(P<0.017)$.

\section{DISCUSSION}

This study compared the effectiveness of US-guided versus blind GNB in the treatment of knee OA based on pain, muscle strength with an isokinetic device, physical function, and quality of life. The results indicated that both techniques were effective in reducing the symptoms and improving physical function. Although there was not a significant difference in pain and muscle strength between the groups, participants injected using US performed significantly better during the 30 -s CST and 6MWT from the baseline to the first month compared to the participants injected using the blind technique. Blind injection was more significantly effective on NHP pain and emotional reaction subscales after the third month from baseline, and the NHP social isolation subscale after the first month from baseline. Pain during injection was significantly less in the US group. When comparing the first month and third month to baseline, significant recovery was observed in VAS, WOMAC-stiffness, SCT, TUGT, and 30-s CST in the group injected using US. Significant recovery was observed in VAS, WOMAC-physical function, total, and NHP-pain in the blind injection group. In the first month, there was significant improvement in NHP-pain, social isolation, and 6MWT parameters in the US group, and in SCT and TUGT for the blind injection group. At the same time, the third month results of the WOMAC-total in the US group and NHP-emotional reaction and social isolation in the blind injection group were significantly different from the baseline. Although these are good results, significant regression was determined in the SCT, 30-s CST, and 6MWT in the US group, and the VAS, WOMACphysical function, SCT, and 6MWT in the blind injection group between the first and third month. No effect of GNB was determined on isokinetic muscle strength.

GNB can be performed with different imaging devices, but US is the most commonly used imaging device with the aim of increasing procedure accuracy. The genicular nerves mostly travel near the superior medial, lateral, and inferior medial arteries, which are easily identified by color Doppler at the junction of the of the epiphysis with the shafts of the femur and tibia [16]. We performed US-guided injection with this technique. However, previous studies haven't compared the blind injection technique of the GNB with other techniques; some cadaveric studies determined some landmarks for the GNB $[10,11]$. After three lines were drawn between the fibular head, femoral medial, and lateral epicondyles, an injection was performed on the superolateral, superomedial, and inferomedial corners of the lines.

Kim et al. [3] compared the efficiency of GNB with local anesthetic with corticosteroids for knee OA. In this study, GNB with local anesthetic judged as effective, and the addition of a corticosteroid didn't make any difference in efficiency [16]. In another study comparing two different imaging methods, both techniques were found to be effective in pain and functionality assessed by WOMAC, and the efficiency was prolonged to three months [3]. Yilmaz et al. [15] showed GNB with intraarticular steroid injection is a treatment for pain intensity and WOMAC, but they didn't find significant improvement in the NHP for knee OA . VAS scores were decreased in first and third months with both two techniques of GNB, and there was no difference between the two techniques. In our study, after GNB with US, we found significant improvement in the WOMAC-total, and GNB without imaging didn't reduce its effectiveness. We observed a significant effect on the NHP pain, emotional reaction, and social isolation subscales in the blind injection group; and we thought that higher baseline scores of the NHP emotional reaction and social isolation in the blind injection group could be an impact on improving this result. Also, the reason for this result in evaluating health-related quality of life may be due to many factors threatening the quality of life, since patients 
with $\mathrm{OA}$ are generally older.

Patients with knee OA have poor functional capacity, so functional tests are very important for these patients. In order to evaluate all aspects of functional limitations, it is recommended to use a combination of tests rather than one test [23]. The effectiveness of the GNB on functional tests has never been investigated until our study. In addition, we used the TUGT, 30-s CST, 6MWT, and SCT to evaluate the functionality. When we compared the two techniques, we found greater improvement in the 6MWT and 30-s CST in the US group than in the blind injection group in the first month. According to first-month results, significant improvement was determined for all functional tests in the US group. Although the performance of the participants in the 6MWT, SCT, and 30-s CST significantly decreased between the first month and third month, the SCT, TUGT, and 30-s CST had significantly better results at the end of the third month than at baseline. On the other hand, only the SCT and TUGT results were significantly better at the end of the first month after the blind injection. Therefore, GNB with US might provide significant benefits for functionality when compared to GNB without imaging.

Muscle weakness in the quadriceps and hamstring muscles is commonly seen in patients with knee OA [24]. In a study comparing the effects of intraarticular corticosteroid and hyaluronic acid injections on muscle strength, it was found that intraarticular hyaluronic acid injection is an effective treatment for improving isokinetic muscle strength. In addition, its effect lasted six months. This result provided the patients better performance in rehabilitation protocol with the decreasing pain after injection [15]. In our study we didn't find any effect from GNB on isokinetic muscle strength. Since the effect of the GNB is short-lived, this could explain the inability to increase muscle strength.

In our study, no side effects were encountered in either group during the three months. The participants felt less pain during injection, and suprapatellar effusion was detected in five patients with sonographic evaluation in the US group. Overall, it can be said that GNB with US is useful to show any concomitant pathology, and make the patient more comfortable.

This study has some limitations; the first limitation was the patients injected with the blind technique weren't evaluated with US because blind injection was performed by another observer. Therefore, some pathologies might have been overlooked. Another limitation of our study was that the patients were shown the rehabilitation protocol before injection, but whether the patient applied the rehabilitation protocol appropriately was questioned only for the first and third months. If the rehabilitation protocols could be checked more frequently, the compliance with the rehabilitation program might have been increased, and an isokinetic muscle strength increase might have been detected. The last limitation of our study was that we didn't compare and evaluate how much time we spent on each protocol. If we had measured these parameters, it may be helpful for other practitioners when choosing the protocol.

Despite these limitations, we believe that this is a strong work. The main strength of this research is that it is the first study which evaluates the GNB on muscle function performed using the isokinetic dynamometer. In our study, we tested functional level with not only a questionnaire but also with functional clinical tests. Another difference of our study to other studies is that we are the first to compare the effectiveness of US-guided and blind GNB.

Consequently, according to our results, no difference was found between US-guided and blind GNB on pain, functionality according to WOMAC, and isokinetic hamstring and quadriceps muscle functions. On the other hand, since patients experienced less pain during USguided injection and additional pathologies could be detected, it can be said that the US-guided injection is more comfortable.

\section{CONFLICT OF INTEREST}

No potential conflict of interest relevant to this article was reported.

\section{FUNDING}

No funding to declare.

\section{ORCID}

Damla Cankurtaran, https://orcid.org/0000-0002-6208-3345

Ozgur Zeliha Karaahmet, https://orcid.org/0000-0002-1338-8935

Sadik Yigit Yildiz, https://orcid.org/0000-0002-3939-9835

Emel Eksioglu, https://orcid.org/0000-0002-4682-3833

Deniz Dulgeroglu, https://orcid.org/0000-0003-2491-1717

Ece Unlu, https://orcid.org/0000-0001-5590-5075

\section{REFERENCES}

1. Li CF, Chen YJ, Lin TY, Hsiao YH, Fu JC, Chen CH, et al. Immediate responses of multi-focal low level laser therapy on quadriceps in knee osteoarthritis patients. Kaohsiung J Med 
Sci 2019; 35: 702-7.

2. Dass RM, Kim E, Kim HK, Lee JY, Lee HJ, Rhee SJ. Alcohol neurolysis of genicular nerve for chronic knee pain. Korean J Pain 2019; 32: 223-7.

3. Kim DH, Lee MS, Lee S, Yoon SH, Shin JW, Choi SS. A prospective randomized comparison of the efficacy of ultrasound- vs fluoroscopy-guided genicular nerve block for chronic knee osteoarthritis. Pain Physician 2019; 22: 139-46.

4. Crawford DC, Miller LE, Block JE. Conservative management of symptomatic knee osteoarthritis: a flawed strategy? Orthop Rev (Pavia) 2013; 5: e2.

5. Santaguida PL, Hawker GA, Hudak PL, Glazier R, Mahomed NN, Kreder HJ, et al. Patient characteristics affecting the prognosis of total hip and knee joint arthroplasty: a systematic review. Can J Surg 2008; 51: 428-36.

6. Kesikburun S, Yașar E, Uran A, Adigüzel E, Yilmaz B. Ultrasound-guided genicular nerve pulsed radiofrequency treatment for painful knee osteoarthritis: a preliminary report. Pain Physician 2016; 19: E751-9.

7. Choi WJ, Hwang SJ, Song JG, Leem JG, Kang YU, Park PH, et al. Radiofrequency treatment relieves chronic knee osteoarthritis pain: a double-blind randomized controlled trial. Pain 2011; 152: 481-7.

8. McCormick ZL, Korn M, Reddy R, Marcolina A, Dayanim D, Mattie $\mathrm{R}$, et al. Cooled radiofrequency ablation of the genicular nerves for chronic pain due to knee osteoarthritis: sixmonth outcomes. Pain Med 2017; 18: 1631-41.

9. Protzman NM, Gyi J, Malhotra AD, Kooch JE. Examining the feasibility of radiofrequency treatment for chronic knee pain after total knee arthroplasty. PM R 2014; 6: 373-6.

10. Yasar E, Kesikburun S, Kılıç C, Güzelküçük Ü, Yazar F, Tan AK. Accuracy of ultrasound-guided genicular nerve block: a cadaveric study. Pain Physician 2015; 18: E899-904.

11. Sutaria RG, Lee SW, Kim SY, Howe R, Downie SA. Localization of the lateral retinacular nerve for diagnostic and therapeutic nerve block for lateral knee pain: a cadaveric study. PM R 2017; 9: 149-53.

12. Baert IAC, Meeus M, Mahmoudian A, Luyten FP, Nijs J, Verschueren SMP. Do psychosocial factors predict muscle strength, pain, or physical performance in patients with knee osteoarthritis? J Clin Rheumatol 2017; 23: 308-16.

13. Altman R, Asch E, Bloch D, Bole G, Borenstein D, Brandt K, et al. Development of criteria for the classification and reporting of osteoarthritis. Classification of osteoarthritis of the knee. Diagnostic and Therapeutic Criteria Committee of the American Rheumatism Association. Arthritis Rheum 1986; 29: $1039-49$.

14. Kellgren JH, Lawrence JS. Radiological assessment of osteo- arthrosis. Ann Rheum Dis 1957; 16: 494-502.

15. Yilmaz V, Umay E, Gundogdu I, Aras B. The comparison of efficacy of single intraarticular steroid injection versus the combination of genicular nerve block and intraarticular steroid injection in patients with knee osteoarthritis: a randomised study. Musculoskelet Surg 2019. doi: 10.1007/ s12306-019-00633-y.

16. Bellamy N, Buchanan WW, Goldsmith CH, Campbell J, Stitt LW. Validation study of WOMAC: a health status instrument for measuring clinically important patient relevant outcomes to antirheumatic drug therapy in patients with osteoarthritis of the hip or knee. J Rheumatol 1988; 15: 1833-40.

17. Busija L, Pausenberger E, Haines TP, Haymes S, Buchbinder $\mathrm{R}$, Osborne RH. Adult measures of general health and healthrelated quality of life: Medical Outcomes Study Short Form 36-Item (SF-36) and Short Form 12-Item (SF-12) Health Surveys, Nottingham Health Profile (NHP), Sickness Impact Profile (SIP), Medical Outcomes Study Short Form 6D (SF6D), Health Utilities Index Mark 3 (HUI3), Quality of WellBeing Scale (QWB), and Assessment of Quality of Life (AQoL). Arthritis Care Res (Hoboken) 2011; 63 Suppl 11: S383-412.

18. Podsiadlo D, Richardson S. The timed "Up \& Go": a test of basic functional mobility for frail elderly persons. J Am Geriatr Soc 1991; 39: 142-8.

19. Guyatt GH, Sullivan MJ, Thompson PJ, Fallen EL, Pugsley SO, Taylor DW, et al. The 6-minute walk: a new measure of exercise capacity in patients with chronic heart failure. Can Med Assoc J 1985; 132: 919-23.

20. Jones CJ, Rikli RE, Beam WC. A 30-s chair-stand test as a measure of lower body strength in community-residing older adults. Res Q Exerc Sport 1999; 70: 113-9.

21. Norman GR, Sloan JA, Wyrwich KW. Interpretation of changes in health-related quality of life: the remarkable universality of half a standard deviation. Med Care 2003; 41: 582-92.

22. Gkrilias P, Tsepis E, Kokkalis Z, Panagiotopoulos E, Megas P. The relationship between isokinetic strength and functional performance tests in patients with knee osteoarthritis. J Phys Ther Sci 2018; 30: 888-91.

23. Kim DH, Choi SS, Yoon SH, Lee SH, Seo DK, Lee IG, et al. Ultrasound-guided genicular nerve block for knee osteoarthritis: a double-blind, randomized controlled trial of local anesthetic alone or in combination with corticosteroid. Pain Physician 2018; 21: 41-52.

24. Maia PAV, Cossich VRA, Salles-Neto JI, Aguiar DP, de Sousa EB. Viscosupplementation improves pain, function and muscle strength, but not proprioception, in patients with knee osteoarthritis: a prospective randomized trial. Clinics (Sao Paulo) 2019; 74: el207. 